\title{
Crosslinked Carbon Nano Dots Membranes for Organic Solvent Nanofiltration
}

\author{
Mahsa Abbaszadeh, Andrew Atkinson, Kevin Trinh, and Santanu Kundu* \\ Dave C Swalm School of Chemical Engineering, Mississippi State University, MS 39762, USA \\ E-mail: santanukundu@che.msstate.edu
}

Keywords: free-standing nanofilms, carbon nano dots, thin-film composite (TFC) membranes, dye removal, layer-by-layer assembly 


\begin{abstract}
The development of custom-designed membranes with high selectivity is vital for their usage in organic solvent nanofiltration (OSN). Here, we report an OSN membrane with an active layer built using carbon nano dots (CNDs). A layer-by-layer (LbL) framework was used in obtaining a thin, hybrid (organic-inorganic) active layer by covalently crosslinking trimesoyl chloride and aminefunctionalized CNDs. Because of modularity, the LbL technique allows the fabrication of an active layer on a substrate of choice, including silicon wafer, and polyacrylonitrile (PAN). Nanofilms formed on the silicon wafer has been rendered free-standing by dissolving a sacrificial interlayer. These free-standing nanofilms are mechanically stable, can withstand exposure to organic solvents, and can be transferred readily onto a porous substrate of interest, such as PAN, to form a thin film composite (TFC) membrane. The TFC membranes display tight molecular weight cutoff, such as $\sim 90 \%$ rejection for dispersed red dye with a molecular weight of $314.34 \mathrm{~g} \mathrm{~mol}^{-1}$ in methanol. Taking advantage of the distinct fluorescence properties of CNDs and the films built with those, a crack in the film can be easily detected. This property can be harnessed for diagnostic purposes, such as tracking mechanical failure and fouling of membrane active layer.
\end{abstract}




\section{Introduction}

Commercial membranes with different pore sizes are already being used for many size-selective separations applications, ranging from water desalination through reverse osmosis process to bioseparation, and many new applications have been envisioned. ${ }^{1-4}$ Specifically, organic solvent nanofiltration (OSN) is an emerging area of application because of its promise of lower energy consumption in comparison to other separation methods such as distillation. ${ }^{5,6}$ As the OSN process typically involves smaller organic molecules, a dense network with a small pore size is necessary. Such a dense network can be found in commercially available thin-film composite (TFC) membranes, for example, polyamide membranes, which have been investigated for the OSN applications. ${ }^{7,8}$ However, the interactions between the organic solvents and the membrane active layer and porous support leading to swelling and degradation pose challenges for the rapid deployment of commercially available reverse osmosis and nanofiltration membranes for OSN applications. ${ }^{9}$ Thus, the development of custom membranes is necessary by introducing new chemistries for the active layer, which then can be combined with a suitable support layer. ${ }^{10}$ Among many strategies, hybrid active layers consisting of polymers and inorganic nanomaterials are being investigated, as these can have better mechanical properties and can also be rendered compatible with a wide range of organic solvents. ${ }^{11-13}$

Here, we report a hybrid, organic-inorganic membrane active layer obtained by crosslinking functionalized carbon nano dots (CNDs) and trifunctional acyl chloride, viz., trimesoyl chloride. To synthesize the active layer a layer-by-layer $(\mathrm{LbL})$ technique has been adopted. Because of the modularity and flexibility, the LbL process can facilitate the development and optimization of new chemistry and improvement of the established chemistry toward better performing TFC membrane active layers. ${ }^{14-18}$ Further, the LbL strategy allows one to prepare membrane active layers on any 
substrate of choice, which then can be made free-standing, if necessary. In our case, we have synthesized the active layer on hydrolyzed PAN (HPAN) substrate in obtaining a functional membrane. In addition, a mechanically stable, free-standing nanofilm was also obtained and was transferred on an HPAN substrate. In both cases, we have shown that the active layer can efficiently separate organic dye molecules from various organic solvents. We have considered HPAN as the support layer, as it has adequate chemical stability to withstand the organic solvents used in the fabrication method and filtration tests. ${ }^{19,20}$

Nanomaterials such as graphene and graphene oxide nanoplatelets (GNPs and GONPs) and carbon nanotubes (CNTs) are increasingly being incorporated in various membranes. These can improve membrane performances and, at the same time, render additional functionalities, for example, enhanced fouling and chlorine resistance of graphene containing desalination membranes. ${ }^{21-24}$ Although beneficial, GNPs, GNOPs, and CNTs are much larger than the pore sizes, therefore, precise control of membrane nanostructure is difficult. To circumvent this, we consider spherical CNDs having dimensions much smaller than other carbon nanomaterials enabling further control of the membrane structure and properties. ${ }^{25-27}$ In addition, CNDs are attractive because of their low-cost synthesis method, facile functionalization, aqueous solubility, photoluminescent properties, and low toxicity. ${ }^{28-31}$

\section{Results and Discussion}

CNDs were synthesized here by using a solvothermal synthesis method from mphenylenediamine (MPD), where MPD in ethanol was heated at $180^{\circ} \mathrm{C}$ for 12 hours inside a PTFE lined autoclave (Figure 1a). ${ }^{32,33}$ This method allows us to synthesize a large quantity of CNDs in an inexpensive one-step process. ${ }^{25}$ The synthesized CNDs are decorated with amine functional groups. Because of the amine functional groups, the CNDs can react with the highly reactive acyl- 
chloride groups in TMC, leading to the formation of a three dimensional tightly crosslinked network structure. The CNDs synthesized here are photoluminescent and the bright cyan color of CNDs solution under UV light is shown in (Figure 1b). This fluorescence nature of CNDs is beneficial for diagnostic purposes, particularly for crack determination, and capturing the level of fouling in membranes.

To characterize the CNDs further, TEM was used (Figure 1c) and spherical morphology of synthesized CNDs is evident from a larger CND particle shown as an inset in Figure 1c. The synthesized CNDs are of the order of $10 \mathrm{~nm}$, as captured by TEM.

Figure 1d illustrates a schematic of CNDs thin film synthesis by using a layer-by-layer (LbL) framework by covalently crosslinking trimesoyl chloride and CNDs. The LbL fabrication has been conducted either on a silicon wafer with a polyacrylic acid (PAA) layer or HPAN support with a polyelectrolyte (PE) layer. The PAA and PE layers have been considered as mid-layer or interlayer, as those are sandwiched in between the active layer and the substrate. Three layers of PAA was spun coated on a UVO treated silicon wafer using a $17.5 \mathrm{wt} . \%$ aqueous PAA solution. The CNDs containing nanofilm was synthesized on top of the PAA layer by alternatively depositing CNDs and TMC solutions in toluene. Acetone and toluene were used in the wash steps after the deposition of CNDs and TMC, respectively. ${ }^{34}$ The acyl chloride functional groups of trimesoyl chloride react with the amine functional groups of CNDs. Subsequent depositions of CNDs, acetone, TMC, and toluene resulted in one layer. Here, we conducted our investigations on the films consisting of 30 such layers.

After the required numbers of layers are formed, the silicon wafer with the synthesized film was immersed in a water bath. ${ }^{35}$ The PAA layer dissolved in water and CNDs nanofilm become floating on water (Figure 1e, Figure S1). This free-standing nanofilm is mechanically stable and can be 
transferred on a lasso (Figure 1f) or on HPAN substrate for OSN applications. Transferred freestanding CNDs nanofilm on HPAN is called FS-CNDs throughout the text.

a)
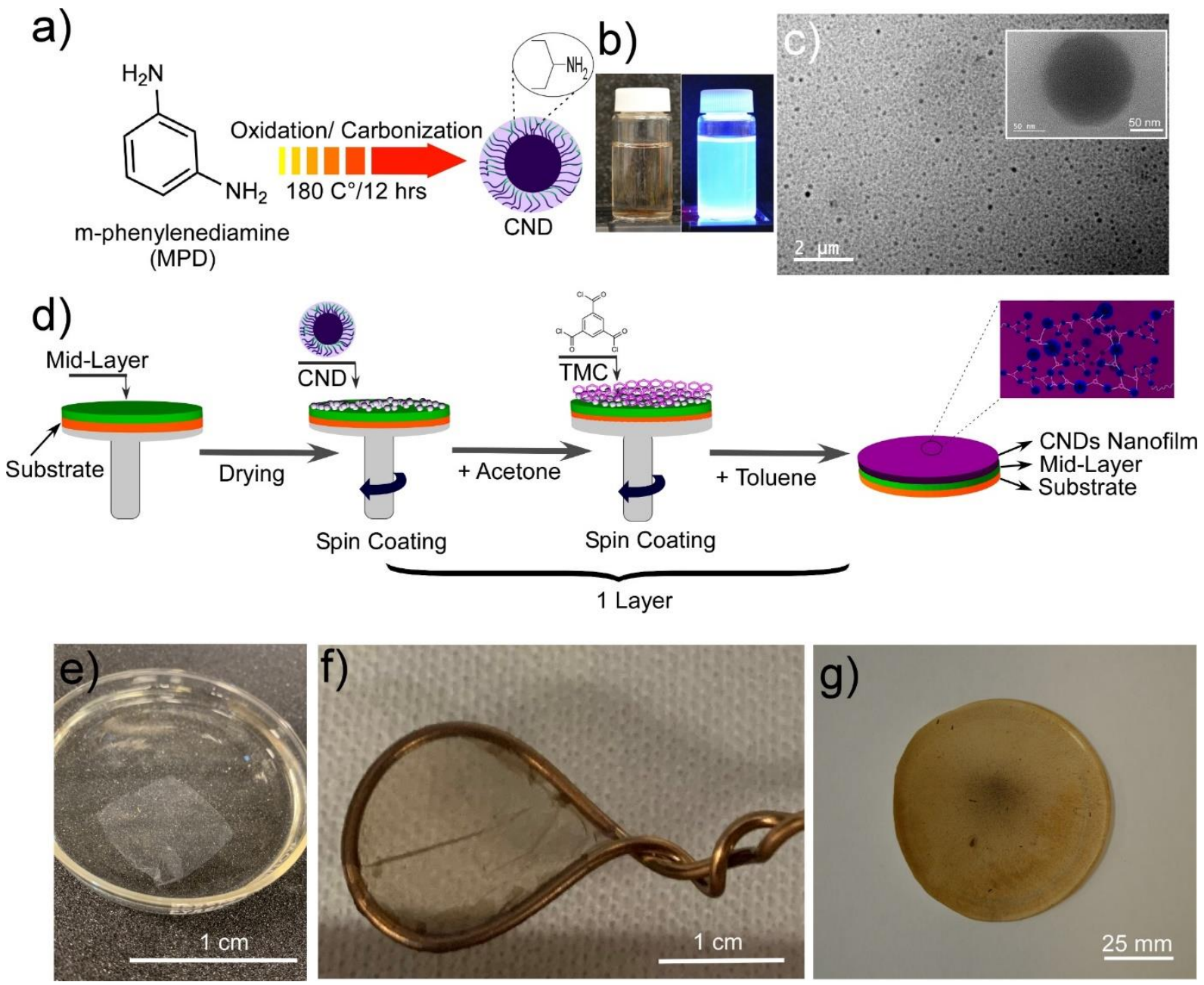

Figure 1. Synthesis of carbon nano dots (CNDs) and crosslinked thin films. a) Schematic of CNDs synthesis; b) Image of synthesized CNDs solution under regular and UV (wavelength: $365 \mathrm{~nm}$ ) lights; c) TEM image of CNDs; d) Magnified TEM image of one CND; e) Schematic of layer-bylayer synthesis process of thin films where CNDs are connected by trimesoyl chloride (TMC). Layers of CNDs and TMC were deposited sequentially on a substrate via spin coating. Acetone and toluene were also used to wash the excess reactants. e) Free-standing CNDs nanofilm floating on water; g) Freestanding CNDs nanofilm on a lasso; h) Thin film fabricated on a HPAN support (DF-CNDs) for separation application. 
For the direct formation of crosslinked CNDs films on HPAN, a polyelectrolytes (PE) layer was deposited on the HPAN surface. The fabrication of the PE layers involves the alternative deposition of polyethyleneimine (PEI) and polyacrylic acid (PAA) aqueous solutions. ${ }^{22}$ The PE layer covered the porous HPAN rough surface, prevented the monomer solutions from penetrating into the porous substrate during the LbL process. ${ }^{34}$ Further, this layer provides good adhesion between the thin film and the substrate via electrostatic forces. The PE layer is relatively permeable and does not attribute to separation. ${ }^{35}$ The directly formed CNDs thin film on HPAN containing PE layer is called DF-CNDs throughout the text. The DF-DNDs has been directly investigated for OSN applications (Figure 1g).

The microstructure of the LbL-assembled CNDs thin films is shown in Figure 2. The scanning electron microscopy (SEM) image of free-standing nanofilm presented in Figure 2a captures the film thickness of $\approx 150 \mathrm{~nm}$. The mechanical robustness of the free-standing nanofilms allows us to conduct the SEM on this sample. The film surface appears to be smooth (Figure 2b). Further, the morphology of free-standing CNDs nanofilms was investigated using TEM, and the presence of spherical CNDs in the nanofilm has been captured clearly (Figure 2c).

Atomic force microscopy (AFM) was further used to verify the sample thickness and to investigate the surface morphology of the nanofilm. Figure 2d, e presents AFM image of 30-layer nanofilm on a silicon wafer where a portion of the film was removed by scratching with a razor. The difference in the heights between the bare wafer and nanofilm provides a thickness of $\approx 150$ $\mathrm{nm}$, similar to that obtained using SEM (Figure S2). Figure 2f displays the 3D AFM image of the sample, capturing the sample roughness at the nanoscale, which could not be captured using SEM From the AFM height image of CNDs nanofilm, the surface roughness has been estimated as Rq $\approx 72.7 \mathrm{~nm}(\mathrm{Ra} \approx 58.9 \mathrm{~nm}, \mathrm{Rmax} \approx 294.0 \mathrm{~nm})$. Due to the variation of CNDs sizes and possible 
agglomerations of carbon nano dots on the surface of nanofilm, the surface smoothness was rather higher than the other LbL synthesized films, as reported previously for LbL synthesized polyamide films. $^{22}$
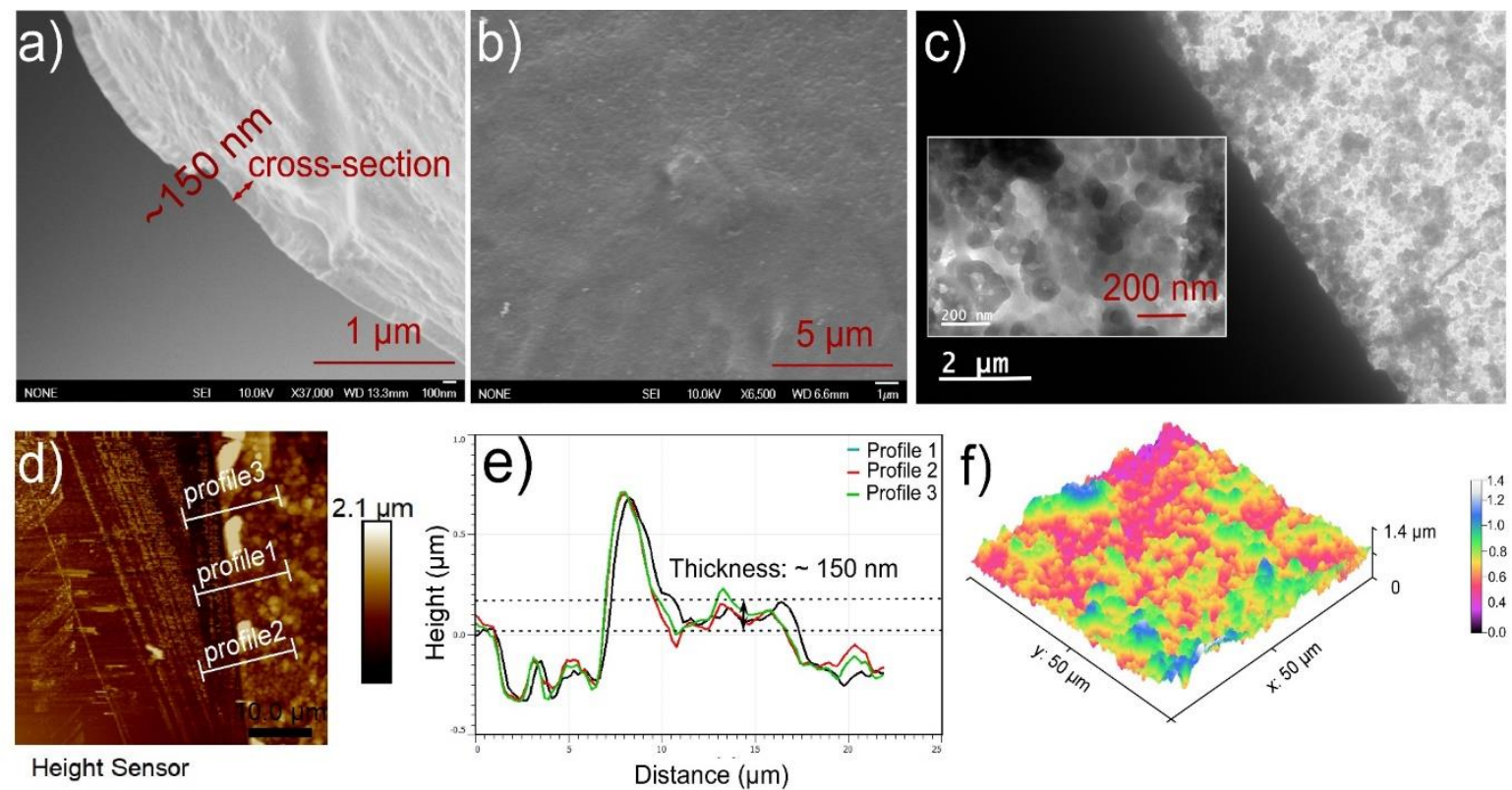

Height Sensor
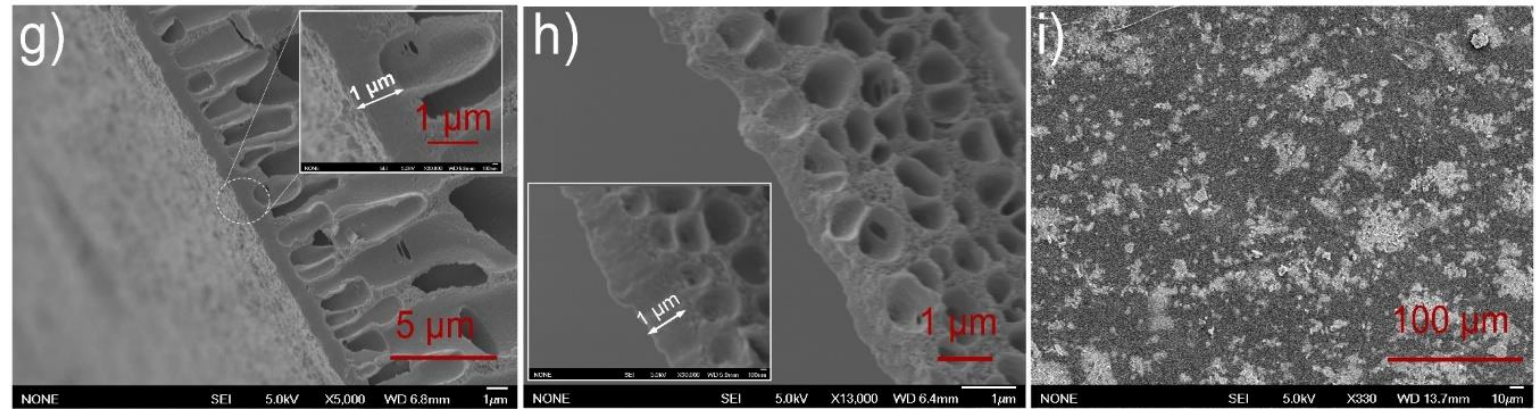

Figure 2. CNDs thin film microstructure. a) SEM of free-standing CNDs nanofilm cross-section; b) SEM of free-standing CNDs nanofilm surface; c) TEM of free-standing CNDs nanofilm transferred on carbon grid (the inset shows the zoomed image); d, e) Thickness measurements of CNDs nanofilm synthesized on silicon wafer acquired using AFM. The sample was scratched via a razor blade and difference in heights between the bare wafer and nanofilm surface is considered as the sample thickness (e); f) AFM 3D height profile of CNDs nanofilm on a silicon wafer; g) SEM cross-sectional view of DF-CNDs (the inset shows the zoom-in image); h) SEM of backside 
of a pealed CNDs thin film from DF-CNDs substrate (the inset shows the zoom-in view); i) SEM surface image of DF-CNDs.

Figure 2g shows the SEM image of DF-CNDs, and the thickness of CNDs thin film has been estimated to be $\approx 1 \mu \mathrm{m}$. Further, the CNDs thin film was peeled from the HPAN/PE substrate, and a SEM image of the backside of the thin film (Figure $2 \mathrm{~h}$ ) captures an imprint of the porous HPAN structure. Figure $2 \mathrm{i}$ shows the SEM surface morphology of DF-CNDs. We hypothesize that the smoothness and homogeneity of the substrate play an important role on the thickness growth for the LbL process. Since the surface of the silicon wafer with PAA layer is considerably smoother than that on the HPAN/PE, the deposition of CNDs and TMC was more even. Further, less aggregation of CNDs and the associated entrapment of reactants led to a thinner film.

The contact angle of CNDs nanofilm is measured (Figure S3) as $50 \pm 2^{\circ}$, indicating the hydrophilic nature of the nanofilm. This hydrophilicity of our nanofilm can be related to the amine groups of the CNDs. ${ }^{36}$ X-ray photoelectron spectroscopy (XPS) was utilized to capture the composition of CNDs and CNDs containing nanofilms. The long scan XPS spectrum of CNDs powder shows three distinct peaks at $285.0 \mathrm{eV}$ (C1s, 73.4\%), $399.3 \mathrm{eV}$ (N1s, 19.1\%), and 532.1 $\mathrm{eV}(\mathrm{O} 1 \mathrm{~s}, 6.8 \%)$. The $\mathrm{C} 1 \mathrm{~s}$ deconvolution of CNDs display three distinct peaks at $284.1 \mathrm{eV}(\mathrm{C}-\mathrm{C} /-$ C-H), $285.3 \mathrm{eV}(-\mathrm{C}-\mathrm{N})$, and $286.5 \mathrm{eV}$ (C-O-H bond) (Figure S4-b). ${ }^{20,24,33,37}$ For CNDs nanofilm, the XPS long scan spectra shows three peaks at $285.1 \mathrm{eV}(\mathrm{C} 1 \mathrm{~s}, 74.9 \%), 400.1 \mathrm{eV}(\mathrm{N} 1 \mathrm{~s}, 12.6 \%)$, and $531.9 \mathrm{eV}(\mathrm{O} 1 \mathrm{~s}, 12.4 \%)$. Upon reaction of amine groups of CNDs with TMC acyl chloride groups and formation of amide bonds, the amount of oxygen increases in the CNDs nanofilm compared to CNDs powder. For CNDs nanofilm, the C1s deconvolution of CNDs nanofilm displays three distinct peaks at $284.5 \mathrm{eV}(\mathrm{C}-\mathrm{C} /-\mathrm{CH}), 285.6 \mathrm{eV}(\mathrm{C}-\mathrm{N})$ and $287.9 \mathrm{eV}(\mathrm{O}-\mathrm{C}=\mathrm{O} / \mathrm{O}=\mathrm{C}-$ N) (Figure S4-d). ${ }^{37,38} \mathrm{C} 1 \mathrm{~s}$ deconvolution results of CNDs nanofilm confirms the reaction of TMC 
acyl chlorides to the CNDs amine/hydroxyl functional groups leading to the formation of $\mathrm{O}=\mathrm{C}$ $\mathrm{N} / \mathrm{O}-\mathrm{C}=\mathrm{O}$ groups. This is further investigated using FTIR.

FTIR spectra of the CNDs powder and DF-CNDs membrane over the wavenumbers of 4500$700 \mathrm{~cm}^{-1}$ are presented in Figure S5. In this wavenumber region, the peak at $1582 \mathrm{~cm}^{-1}$ is attributed to the $\mathrm{N}-\mathrm{H}$ stretching vibrations for CNDs powder, confirming the presence of nitrogen-containing functional groups on the CNDs. ${ }^{5,39,40}$ With the formation of CNDs thin film, the FTIR of DF-CNDs shows a band around $1541 \mathrm{~cm}^{-1}$ which is ascribed to the N-H in-plane bending of amide II. ${ }^{41}$ The band around $1606 \mathrm{~cm}^{-1}$ is attributed to the $\mathrm{C}=\mathrm{O}$ stretching vibrations (carbonyl group). ${ }^{39}$ The band around $1657 \mathrm{~cm}^{-1}$ is attributed to the $\mathrm{C}=\mathrm{O}$ stretching vibrations (amide I). ${ }^{22}$ The amide bond formation further supports our conclusions of chemical reactions between TMC with CNDs.

The selectivity and permeability of FS-CNDs and DF-CNDs were tested in a lab-scale dead-end cell system with different organic solvents. For the FS-CNDs, the pure solvent permeance values for four organic solvents, methanol, ethanol, isopropanol, and toluene are summarized in Figure 3a. Methanol has the highest permeance of $\approx 2.3 \mathrm{~L} \mathrm{~m}^{-2} \mathrm{~h}^{-1}$ bar $^{-1}$ followed by ethanol (permeance $\approx$ $1.7 \mathrm{~L} \mathrm{~m}^{-2} \mathrm{~h}^{-1}$ bar $^{-1}$ ), isopropanol (permeance $\approx 0.8 \mathrm{~L} \mathrm{~m}^{-2} \mathrm{~h}^{-1} \mathrm{bar}^{-1}$ ), and toluene (permeance $\approx 0.4 \mathrm{~L}$ $\left.\mathrm{m}^{-2} \mathrm{~h}^{-1} \mathrm{bar}^{-1}\right)$ 

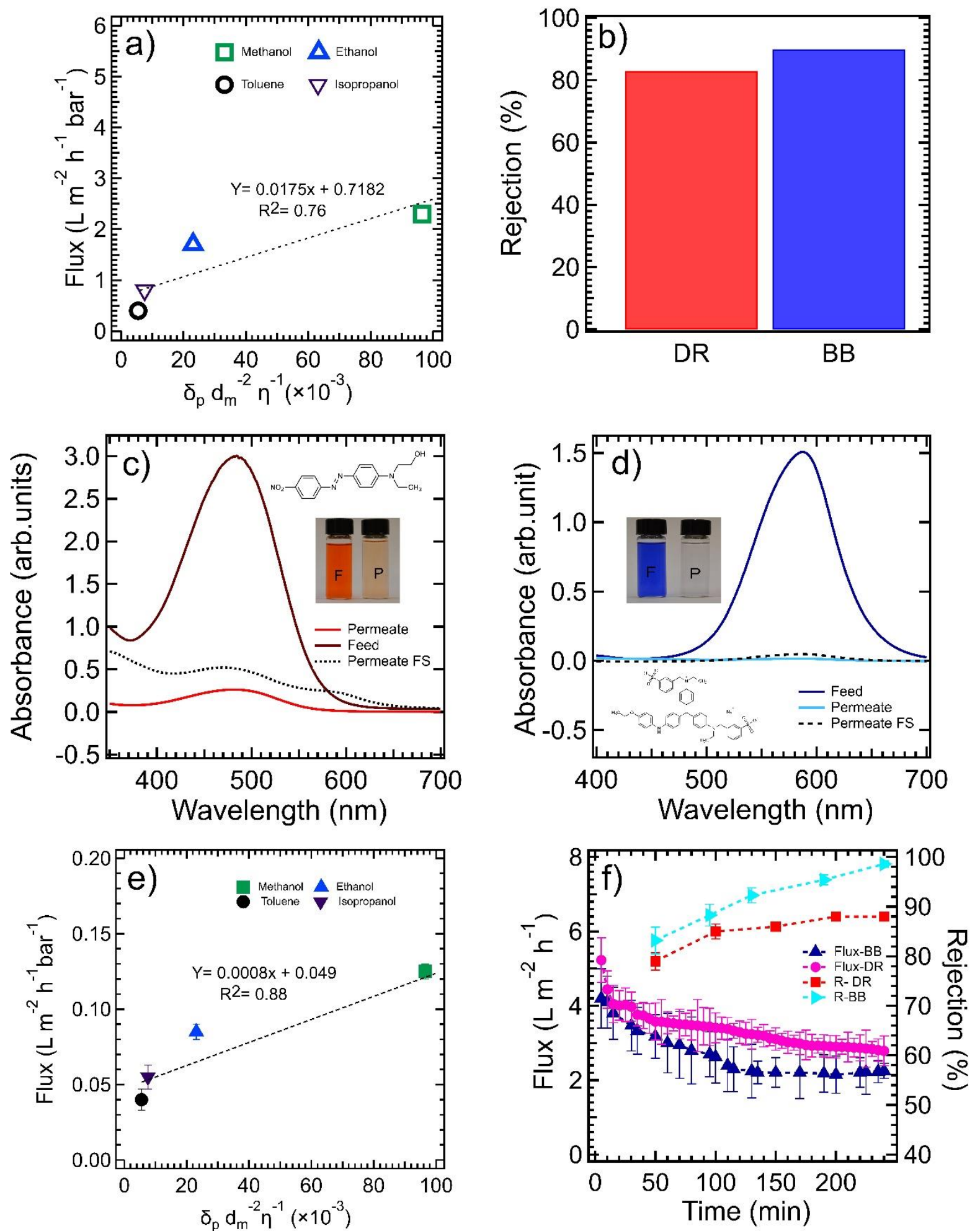

Figure 3. Organic separation performances of CNDs membranes. a) Solvent permeance against the combined solvent properties (viscosity, molar diameter, and solubility parameters) for FS- 
CNDs; b) Dye rejection plot for FS-CNDs. Ultraviolet-visible absorption spectra of c) disperse red 1 (DR), and d) brilliant blue (BB) dye in methanol for feed solutions and permeate. Here, permeate spectra for FS-CNDs is denoted as "permeate FS", whereas, that for the DF-CNDs as "permeate". e) Solvent permeance against the combined solvent properties (viscosity, molar diameter, and solubility parameters) for DF-CNDs; f) Long term flux and rejection values for BB and DR solutions in methanol using DF-CNDs. Pressures of 20 bar was applied for DF-CNDs .

A phenomenological model has been proposed in the literature, where, the permeance scales with $\delta_{p} \eta^{-1} d_{m}^{-2} .{ }^{5}$ We have found that this scaling is also applicable to the FS-CNDs, as shown in Figure 3a. Here, $\delta_{p}$ is the Hansen solubility parameter due to dipole forces, which captures the interaction between the solvent and thin film. $\eta$ is solute viscosity, and $d_{m}$ is the effective molar diameter, capturing size of solvent molecules. ${ }^{19,42}$ Note that although $\delta_{p}$ is the highest for methanol, smaller viscosity and molar diameter attributed to the highest permeance for our case (Table S1). Similarly, higher molar diameter attributes towards lower permeance in toluene.

We have also investigated the separation efficiency of the synthesized thin films using two model dye molecules, brilliant blue R (BB, $825.97 \mathrm{~g} \mathrm{~mol}^{-1}$ ), and disperse red 1 (DR, $314.34 \mathrm{~g} \mathrm{~mol}^{-}$ ${ }^{1}$ ) in methanol. The concentration of dye molecules in the feed (methanol) was fixed at $20 \mathrm{mg} \mathrm{L}^{-1}$. For the FS-CNDs, $90 \%$ and $83 \%$ rejection were achieved for BB and DR, respectively, and such rejection has led to an almost clear permeate solution (Figure 3b). This has been estimated from the UV-vis spectra of the feed (solid line) and permeate (dotted) solutions (Figure 3c, d), and using a calibration plot generated for various dye concentration in methanol. The larger volume of BB molecule $\left(2087.1 \AA^{3}\right)$ in comparison to that of DR molecule $\left(905.6 \AA^{3}\right)$ can be attributed to the higher rejection of $\mathrm{BB} .{ }^{5}$ We also hypothesize that the polarity of the dye molecules has an effect on their permeation through the CNDs thin film. Here, BB is negatively charged, whereas DR is 
neutral. The CNDs thin film is likely to have a negative-charged surface due to its hydrophilic nature, therefore repelling the negatively charged $\mathrm{BB}$ molecules from passing through the membrane pores.

For the DF-CNDs, the pure solvent permeance values are shown in Figure 3-e. In comparison to the FS-CNDs, the pure solvent permeance values for these films are lower, which likely due to the higher thickness of the sample, as the thicker layer provides increased diffusion path length. ${ }^{19,43}$ The permeance values also scale with $\delta_{p} \eta^{-1} d_{m}^{-2}$ with a similar slope that is observed for the FSCNDs.

To investigate the durability of these thin films, we captured the permeability and selectivity of the DF-CNDs for BB and RR dyes in methanol for more than 200 min (Figure 3f). The permeance decreased slightly, and the rejection increased with time, likely due to the compaction of the film. The rejection plateaued at about $98 \%$ for BB (Figure 3f) and $89 \%$ for DR dyes (Figure S6). Reasonably good rejection behavior for DR molecule indicates tight molecular weight cut off. In addition, the DF-CNDs membranes were also tested in a dead-end cell with aqueous $\mathrm{CaCl}_{2}$ and $\mathrm{Na}_{2} \mathrm{SO}_{4}$ solutions (salt concentration $20 \mathrm{mg} / \mathrm{L}$ ) to further investigate the rejection behavior with respect to the solute molecular weight. The DF-CNDs membrane displayed approximately $60.5 \%$ rejection for $\mathrm{CaCl}_{2}$ and $67.3 \%$ for $\mathrm{Na}_{2} \mathrm{SO}_{4}$. Based on the rejection data for different molecular weight materials (Figure S8), the molecular weight cut off (MWCO), defined as the solute molecular weight displaying approximately $90 \%$ rejection, is estimated to be $\sim 320 \mathrm{~g} \mathrm{~mol}^{-1}$

The rejection values for $\mathrm{BB}$ and $\mathrm{DR}$ dyes are a little higher than the transferred free-standing films. However, both of these films display better long-term performance behavior in comparison to the commercial polyamide membranes (for example, SW30HR, Figure S7) ${ }^{44-46}$ Also note that 
the selectivity of thin films synthesized here is comparable or better, even for low molecular weight DR, than polyamide-based membranes (with or without DMF) reported in the literature. ${ }^{44-46}$

The synthesized CNDs are fluorescent active, exhibiting emission maxima at $\approx 455 \mathrm{~nm}$ correspond to blue color. ${ }^{32}$ The fluorescent activity of synthesized CNDs is likely due to their $\mathrm{sp}^{2}$ $\pi$-conjugated structure. ${ }^{42}$ Figure 4 a displays the UV-vis absorption spectra for CNDs solution and thin film where a slight shift in absorption peak for CNDs thin film is visible.

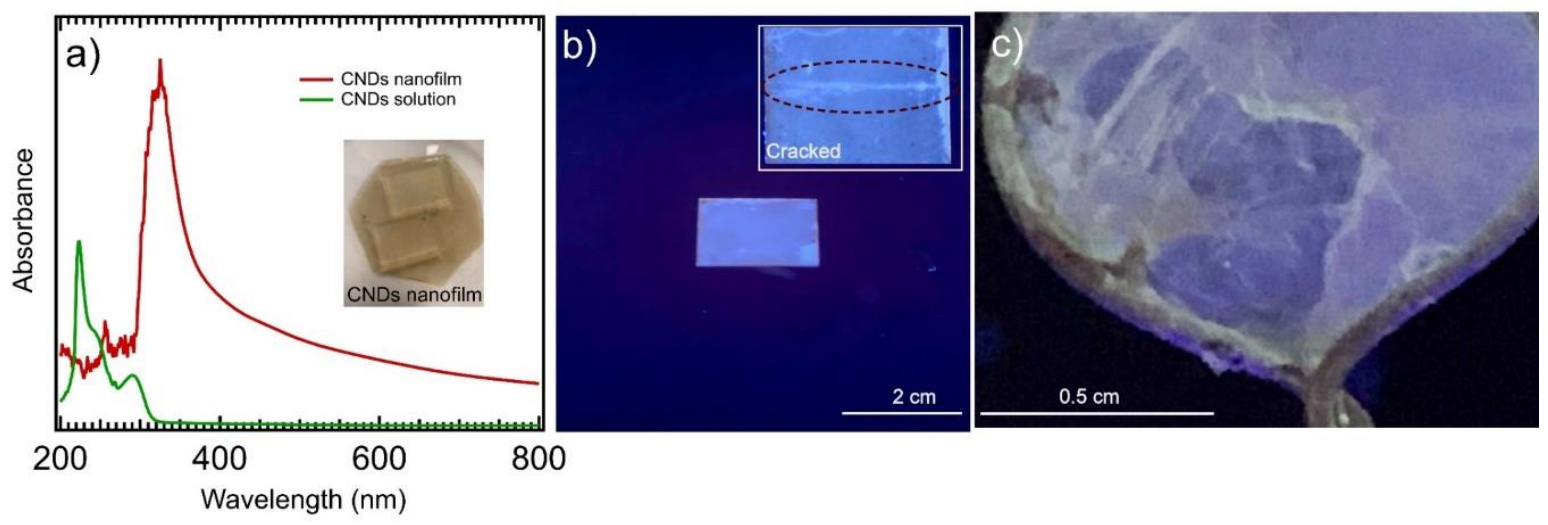

Figure 4. Harnessing fluorescence properties of CNDs films. a) UV-Vis absorption spectra for CNDs solution $(0.1 \mathrm{~g} / \mathrm{L})$ in ethanol and CNDs nanofilm; b) CNDs nanofilm (on a quartz slide) under UV light. The top-right inset shows distinct crack in the nanofilm; c) The free-standing CNDs nanofilm on a lasso under UV light capturing the defects, folding and ruptures.

This can be due to the cross-linking of CNDs with TMC and further suggest the reaction between CNDs and TMC. As the CNDs synthesized here are fluorescent under UV light, the resultant thin film is also fluorescent and the luminescent blue color is clearly observed in Figure 4b. Here, the film is supported on a quartz plate and a free-standing film is shown in Figure S9. A crack was introduced on the supported nanofilm using a razor blade and can be detected easily (Figure 4b). Figure $4 \mathrm{c}$ shows the nanofilm on a lasso under UV light, and the fluorescent property of the nanofilm allows one to recognize the presence of defects, ruptures, or cracks in the nanofilm. The 
ability to detect the cracks and defects on nanofilm via UV light can introduce a new protocol for identifying mechanical fractures or defects in nanofilms or membranes via measuring their fluorescent property. For practical applications of the proposed membrane, crack and defect formation can be easily captured. Also, CNDs can be incorporated in commercial PA membranes and can be used as diagnostic purposes.

\section{Conclusions}

In summary, fluorescent CNDs synthesized via solvothermal method have been used as novel materials for the fabrication of mechanically stable nanofilms, compatible with organic solvents. The CNDs nanofilms were formed via the LbL method on different substrates and can be rendered free-standing, therefore allowing them to be transferred on a substrate of choice. TFC membranes have been prepared with crosslinked CNDs as active layers, and these membranes display excellent nanofiltration capability with tight molecular weight cutoff. The permeance values for the synthesized membranes are lower compared to literature studies because of the thickness of the membrane. In a future study, possible permeability enhancement of the synthesized membranes will be investigated by optimizing the thickness of the active layer, and by introducing spacer layers. The fluorescent property of the CNDs is beneficial for the detection of mechanical fracture in active layers and can also be incorporated in commercial polyamide membranes for diagnostic purposes. Moreover, the LbL method for the active layer fabrication and the possibility of transfer that active layer on any substrates can be harnessed to develop membranes on many types of substrates, for example, alumina, or ceramic, to minimize the swelling and degradation of polymeric support layer, and to study the solvent impacts on intrinsic properties of fabricated active layers. 


\section{Experimental Section}

Carbon nano dots (CNDs) synthesis. For CNDs synthesis, 5 grams of m-phenylenediamine was dissolved in $500 \mathrm{~mL}$ of ethanol. Then the solution was heated inside a PTFE lined autoclave (Tefic Biotech Co., China) at $180{ }^{\circ} \mathrm{C}$ for 12 hours. ${ }^{32}$ Afterward, the solution was cooled to the ambient temperature. In the next step, the solution was centrifuged (30 minutes, $4000 \mathrm{rpm}$ ) for 4 cycles, and CNDs were collected as supernatant. The collected supernatant (CNDs) was then dried overnight using a freeze drier. The synthesized CNDs were characterized by FTIR, XPS, TEM to investigate the physical and chemical properties.

Preparation of freestanding CNDs nanofilm (FS-CNDs). The freestanding CNDs nanofilm was fabricated via the LbL method. First, a silicon wafer was washed and was sonicated for 5 minutes in acetone, methanol, and isopropanol, sequentially. Then, the silicon wafer was treated by ultraviolet ozone (UVO) for 25 minutes to oxidize the surface of silicon wafers. Aqueous polyacrylic acid solution (PAA, 17.5 wt. \%) was deposited on the wafer using a spin coater at 500 rpm for 45 seconds and then at $2000 \mathrm{rpm}$ for 20 seconds. Then, the sample was placed on a hotplate at $120^{\circ} \mathrm{C}$ for 5 minutes. An air-gun was further used to dry the PAA layer. The water-soluble PAA layer was utilized as a sacrificial layer.

Next, for the fabrication of CNDs nanofilm, 1.0 wt. \% solutions of TMC and 1.0 wt. \% CNDs in toluene were prepared. The CNDs solution was sonicated for 90 min prior to use. First, CNDs solution was deposited using a spin coater. This was followed by a washing step with dry acetone. Next, the TMC solution was deposited, followed by a rinse step with dry toluene. After deposition of the reactants and solvents, a $40 \mathrm{~s}$ wait time was provided and was then spun-coated for 20 seconds at $2000 \mathrm{rpm}$. One layer of the active layer was formed by depositing one cycle of CNDs and TMC along with the wash steps. In this study, 30 layers of the active layer were synthesized. 
After the preparation of the nanofilm on a silicon wafer, the silicon wafer was immersed in water. The sacrificial PAA layer dissolved in water and nanofilm gradually went to the water surface.

\section{Preparation of CNDs films directly on HPAN substrate (DF-CNDs). The DF-CNDs} membrane was fabricated by forming crosslinked CNDs films on the PAN substrate. First, a PAN50 membrane with $14.6 \mathrm{~cm}^{2}$ area was hydrolyzed in $2.0 \mathrm{M} \mathrm{NaOH}$ aqueous solution for $2.0 \mathrm{~h}$ to introduce - $\mathrm{COOH}$ groups on the PAN surface. In the next step, a polyelectrolyte (PE) layer was prepared by the LbL method. The PE layer was formed by depositing cationic and anionic aqueous solutions sequentially. For the cationic solution, an aqueous solution of 0.1 wt $\%$ polyethyleneimine (PEI, molecular weight $\approx 750,000 \mathrm{~g} \mathrm{~mol}^{-1}$ ) which contains $0.5 \mathrm{M} \mathrm{NaCl}(\mathrm{pH}$ $\approx 10.6$ ) was used. For anionic solution, an aqueous solution of 0.1 wt. \% polyacrylic acid (PAA, molecular weight $\left.\approx 100,000 \mathrm{~g} \mathrm{~mol}^{-1}\right)$ which contains $0.5 \mathrm{M} \mathrm{NaCl}(\mathrm{pH} \approx 3.6)$ was used. ${ }^{34}$ DI water was used as a wash step. Three PE layers were formed for this study. The thin film was then formed on the PE layer using the LbL process, as described above (see FS-CNDs preparation section).

Characterization. The morphology and surface properties of nanofilms fabricated were investigated by using a high-resolution SEM, TEM, AFM, and contact angle. The chemical composition of nanofilms was characterized by using XPS and FTIR. Further details regarding the characterization studies can be found in supporting information.

\section{Acknowledgments}

The authors acknowledge the help of Dr. Rooban, Dr. Iwei, and Dr. Rivers from the Institute for Imaging \& Analytical Technologies at Mississippi State University for structural characterization, 
and of Dr. Perez at Integrated Microscopy Center, University of Memphis, for XPS characterizations.

\section{ASSOCIATED CONTENT}

Supporting information provides further details regarding sample synthesis and characterization. The supporting information contains details regarding SEM, TEM, AFM, XPS, FTIR, contact angle, and performance studies. Schematic of freestanding nanofilm fabrication, SEM image of FS-CNDs, contact angle image of nanofilm, XPS data for CNDs powder and nanofilm, OSN performance of membranes, MWCO graph of DF-CNDs, FTIR data for CNDs powder and DFCNDs, OSN performance for commercial membrane (SW30HR), fluorescent image of FS-CNDs under UV light are provided.

\section{AUTHOR INFORMATION}

\section{Corresponding Author}

*Santanu Kundu. E-mail: santanukundu@che.msstate.edu

\section{Author Contributions}

The manuscript was written through contributions of all authors. All authors have given approval to the final version of the manuscript.

\section{Funding Sources}

Authors would like to acknowledge financial support from Dave C Swalm School of Chemical Engineering for this work. 


\section{References}

(1) Shaffer, D. L.; Feldman, K. E.; Chan, E. P.; Stafford, G. R.; Stafford, C. M. Characterizing Salt Permeability in Polyamide Desalination Membranes Using Electrochemical Impedance Spectroscopy. J. Membr. Sci. 2019, 583, 248-257.

(2) Marchetti, P.; Jimenez Solomon, M. F.; Szekely, G.; Livingston, A. G. Molecular Separation with Organic Solvent Nanofiltration: A Critical Review. Chem. Rev. 2014, 114 (21), 10735-10806.

(3) Vendamme, R.; Onoue, S.-Y.; Nakao, A.; Kunitake, T. Robust Free-Standing Nanomembranes of Organic/Inorganic Interpenetrating Networks. Nat. Mater. 2006, 5 (6), 494501.

(4) Green, E.; Fullwood, E.; Selden, J.; Zharov, I. Functional Membranes via Nanoparticle Self-Assembly. Chem. Commun. 2015, 51 (37), 7770-7780.

(5) Karan, S.; Jiang, Z.; Livingston, A. G. Sub-10 Nm Polyamide Nanofilms with Ultrafast Solvent Transport for Molecular Separation. Science 2015, 348 (6241), 1347-1351.

(6) Hermans, S.; Mariën, H.; Van Goethem, C.; Vankelecom, I. F. Recent Developments in Thin Film (Nano)Composite Membranes for Solvent Resistant Nanofiltration. Curr. Opin. Chem. Eng. 2015, 8, 45-54.

(7) See-Toh, Y. H.; Ferreira, F. C.; Livingston, A. G. The Influence of Membrane Formation Parameters on the Functional Performance of Organic Solvent Nanofiltration Membranes. $J$. Membr. Sci. 2007, 299 (1), 236-250. 
(8) Jimenez-Solomon, M. F.; Song, Q.; Jelfs, K. E.; Munoz-Ibanez, M.; Livingston, A. G. Polymer Nanofilms with Enhanced Microporosity by Interfacial Polymerization. Nat. Mater. 2016, 15 (7), 760-767.

(9) Jimenez Solomon, M. F.; Bhole, Y.; Livingston, A. G. High Flux Hydrophobic Membranes for Organic Solvent Nanofiltration (OSN) - Interfacial Polymerization, Surface Modification and Solvent Activation. J. Membr. Sci. 2013, 434, 193-203.

(10) Peyravi, M.; Rahimpour, A.; Jahanshahi, M. Thin Film Composite Membranes with Modified Polysulfone Supports for Organic Solvent Nanofiltration. J. Membr. Sci. 2012, 423-424, 225-237.

(11) Lively, R. P.; Sholl, D. S. From Water to Organics in Membrane Separations. Nat. Mater. 2017, $16(3), 276-279$.

(12) Zhao, J.; Zhu, Y.; Pan, F.; He, G.; Fang, C.; Cao, K.; Xing, R.; Jiang, Z. Fabricating Graphene Oxide-Based Ultrathin Hybrid Membrane for Pervaporation Dehydration via Layer-byLayer Self-Assembly Driven by Multiple Interactions. J. Membr. Sci. 2015, 487, 162-172.

(13) Parthiban, V.; Panda, S. K.; Sahu, A. K. Highly Fluorescent Carbon Quantum Dots-Nafion as Proton Selective Hybrid Membrane for Direct Methanol Fuel Cells. Electrochimica Acta 2018, $292,855-864$.

(14) Seman, M. N. A.; Khayet, M.; Hilal, N. Nanofiltration Thin-Film Composite Polyester Polyethersulfone-Based Membranes Prepared by Interfacial Polymerization. J. Membr. Sci. 2010, 348 (1), 109-116. 
(15) Xie, W.; Geise, G. M.; Freeman, B. D.; Lee, H.-S.; Byun, G.; McGrath, J. E. Polyamide Interfacial Composite Membranes Prepared from M-Phenylene Diamine, Trimesoyl Chloride and a New Disulfonated Diamine. J. Membr. Sci. 2012, 403-404, 152-161.

(16) An, Q.-F.; Sun, W.-D.; Zhao, Q.; Ji, Y.-L.; Gao, C.-J. Study on a Novel Nanofiltration Membrane Prepared by Interfacial Polymerization with Zwitterionic Amine Monomers. J. Membr. Sci. 2013, 431, 171-179.

(17) Wang, T.; Yang, Y.; Zheng, J.; Zhang, Q.; Zhang, S. A Novel Highly Permeable Positively Charged Nanofiltration Membrane Based on a Nanoporous Hyper-Crosslinked Polyamide Barrier Layer. J. Membr. Sci. 2013, 448, 180-189.

(18) Li, Y.; Su, Y.; Dong, Y.; Zhao, X.; Jiang, Z.; Zhang, R.; Zhao, J. Separation Performance of Thin-Film Composite Nanofiltration Membrane through Interfacial Polymerization Using Different Amine Monomers. Desalination 2014, 333 (1), 59-65.

(19) Kim, J. H.; Moon, S. J.; Park, S. H.; Cook, M.; Livingston, A. G.; Lee, Y. M. A Robust Thin Film Composite Membrane Incorporating Thermally Rearranged Polymer Support for Organic Solvent Nanofiltration and Pressure Retarded Osmosis. J. Membr. Sci. 2018, 550, 322331.

(20) Hajighahremanzadeh, P.; Abbaszadeh, M.; Mousavi, S. A.; Soltanieh, M.; Bakhshi, H. Polyamide/Polyacrylonitrile Thin Film Composites as Forward Osmosis Membranes. J. Appl. Polym. Sci. 2016, 133 (42). 
(21) Ai, M.; Shishatskiy, S.; Wind, J.; Zhang, X.; Nottbohm, C. T.; Mellech, N.; Winter, A.; Vieker, H.; Qiu, J.; Dietz, K.-J.; Gölzhäuser, A.; Beyer, A. Carbon Nanomembranes (CNMs) Supported by Polymer: Mechanics and Gas Permeation. Adv. Mater. 2014, 26 (21), 3421-3426.

(22) Abbaszadeh, M.; Krizak, D.; Kundu, S. Layer-by-Layer Assembly of Graphene Oxide Nanoplatelets Embedded Desalination Membranes with Improved Chlorine Resistance. Desalination 2019, 470, 114116.

(23) Zhou, F.; Tien, H. N.; Dong, Q.; Xu, W. L.; Sengupta, B.; Zha, S.; Jiang, J.; Behera, D.; Li, S.; Yu, M. Novel Carbon-Based Separation Membranes Composed of Integrated Zero- and One-Dimensional Nanomaterials. J. Mater. Chem. A 2020, 8 (3), 1084-1090.

(24) Huang, L.; Li, Y.; Zhou, Q.; Yuan, W.; Shi, G. Graphene Oxide Membranes with Tunable Semipermeability in Organic Solvents. Adv. Mater. 2015, 27 (25), 3797-3802.

(25) Tao, S.; Feng, T.; Zheng, C.; Zhu, S.; Yang, B. Carbonized Polymer Dots: A Brand New Perspective to Recognize Luminescent Carbon-Based Nanomaterials. J. Phys. Chem. Lett. 2019, $10(17), 5182-5188$.

(26) Zhang, Y.; Li, H.; Luo, Y.; Shi, X.; Tian, J.; Sun, X. Poly(m-Phenylenediamine) Nanospheres and Nanorods: Selective Synthesis and Their Application for Multiplex Nucleic Acid Detection. PLOS ONE 2011, 6 (6), e20569.

(27) Kim, T.; Park, B.; Lee, K. M.; Joo, S. H.; Kang, M. S.; Yoo, W. C.; Kwak, S. K.; Kim, B.S. Hydrothermal Synthesis of Composition- and Morphology-Tunable Polyimide-Based Microparticles. ACS Macro Lett. 2018. 
(28) Shen, J.; Zhu, Y.; Yang, X.; Li, C. Graphene Quantum Dots: Emergent Nanolights for Bioimaging, Sensors, Catalysis and Photovoltaic Devices. Chem. Commun. 2012, 48 (31), 36863699.

(29) Ray, S. C.; Saha, A.; Jana, N. R.; Sarkar, R. Fluorescent Carbon Nanoparticles: Synthesis, Characterization, and Bioimaging Application. J. Phys. Chem. C 2009, 113 (43), 18546-18551.

(30) Bourlinos, A. B.; Stassinopoulos, A.; Anglos, D.; Zboril, R.; Karakassides, M.; Giannelis, E. P. Surface Functionalized Carbogenic Quantum Dots. Small 2008, 4 (4), 455-458.

(31) Li, H.; Kang, Z.; Liu, Y.; Lee, S.-T. Carbon Nanodots: Synthesis, Properties and Applications. J. Mater. Chem. 2012, 22 (46), 24230-24253.

(32) Wijayapala, R.; Meysam Hashemnejad, S.; Kundu, S. Carbon Nanodots Crosslinked Photoluminescent Alginate Hydrogels. RSC Adv. 2017, 7 (79), 50389-50395.

(33) Yuan, Z.; Wu, X.; Jiang, Y.; Li, Y.; Huang, J.; Hao, L.; Zhang, J.; Wang, J. Carbon DotsIncorporated Composite Membrane towards Enhanced Organic Solvent Nanofiltration Performance. J. Membr. Sci. 2018, 549, 1-11.

(34) Choi, W.; Gu, J.-E.; Park, S.-H.; Kim, S.; Bang, J.; Baek, K.-Y.; Park, B.; Lee, J. S.; Chan, E. P.; Lee, J.-H. Tailor-Made Polyamide Membranes for Water Desalination. ACS Nano 2015, 9 (1), 345-355.

(35) Dai, J.; Sullivan, D. M.; Bruening, M. L. Ultrathin, Layered Polyamide and Polyimide Coatings on Aluminum. Ind. Eng. Chem. Res. 2000, 39 (10), 3528-3535. 
(36) Veríssimo, S.; Peinemann, K.-V.; Bordado, J. Influence of the Diamine Structure on the Nanofiltration Performance, Surface Morphology and Surface Charge of the Composite Polyamide Membranes. J. Membr. Sci. 2006, 279 (1), 266-275.

(37) Yuan, Y.; Hu, B.; Tong, C.; Bai, Y.; Lü, C. Novel Quaternized Carbon Dots Modified Polysulfone-Based Anion Exchange Membranes with Improved Performance. Int. J. Hydrog. Energy 2019, 44 (39), 22181-22193.

(38) Pérez-Manríquez, L.; Behzad, A. R.; Peinemann, K.-V. Sub-6 Nm Thin Cross-Linked Dopamine Films with High Pressure Stability for Organic Solvent Nanofiltration. Macromol. Mater. Eng. 2016, 301 (12), 1437-1442.

(39) Sun, H.; Wu, P. Tuning the Functional Groups of Carbon Quantum Dots in Thin Film Nanocomposite Membranes for Nanofiltration. J. Membr. Sci. 2018, 564, 394-403.

(40) Aswathy, N. R.; Palai, A. K.; Mohanty, S.; Nayak, S. K. Freestanding Electrically Conducting Flexible Membranes Based on Novel Chitosan/PANI/RGO Nanocomposites. Mater. Lett. 2020, 259, 126777.

(41) Lu, X.; Romero-Vargas Castrillón, S.; Shaffer, D. L.; Ma, J.; Elimelech, M. In Situ Surface Chemical Modification of Thin-Film Composite Forward Osmosis Membranes for Enhanced Organic Fouling Resistance. Environ. Sci. Technol. 2013, 47 (21), 12219-12228.

(42) Huang, L.; Chen, J.; Gao, T.; Zhang, M.; Li, Y.; Dai, L.; Qu, L.; Shi, G. Reduced Graphene Oxide Membranes for Ultrafast Organic Solvent Nanofiltration. Adv. Mater. 2016, 28 (39), 86698674. 
(43) Tian, M.; Qiu, C.; Liao, Y.; Chou, S.; Wang, R. Preparation of Polyamide Thin Film Composite Forward Osmosis Membranes Using Electrospun Polyvinylidene Fluoride (PVDF) Nanofibers as Substrates. Sep. Purif. Technol. 2013, 118, 727-736.

(44) Pérez-Manríquez, L.; Aburabi’e, J.; Neelakanda, P.; Peinemann, K.-V. Cross-Linked PANBased Thin-Film Composite Membranes for Non-Aqueous Nanofiltration. React. Funct. Polym. $2015,86,243-247$.

(45) Abdellah, M. H.; Pérez-Manríquez, L.; Puspasari, T.; Scholes, C. A.; Kentish, S. E.; Peinemann, K.-V. A Catechin/Cellulose Composite Membrane for Organic Solvent Nanofiltration. J. Membr. Sci. 2018, 567, 139-145.

(46) Xia, L.; McCutcheon, J. R. Understanding the Influence of Solvents on the Intrinsic Properties and Performance of Polyamide Thin Film Composite Membranes. Sep. Purif. Technol. $2020,238,116398$.

(47) Yuan, F.; Li, S.; Fan, Z.; Meng, X.; Fan, L.; Yang, S. Shining Carbon Dots: Synthesis and Biomedical and Optoelectronic Applications. Nano Today 2016, 11 (5), 565-586. 
Supporting Information

\section{Crosslinked Carbon Nano Dots Membranes for Organic Solvent Nanofiltration}

Mahsa Abbaszadeh, Andrew Atkinson, Kevin Trinh, and Santanu Kundu*

M. Abbaszadeh, A. Atkinson, K. Trinh, Dr. S. Kundu

Dave C Swalm School of Chemical Engineering, Mississippi State University, MS 39762, USA

E-mail: santanukundu@che.msstate.edu

1. Materials and methods

1.1. Chemicals and materials

The following materials were used as received from Sigma-Aldrich: branched polyethyleneimine (PEI) with a molecular weight of 750,000 $\mathrm{g} \mathrm{mol}^{-1}$, poly(acrylic acid) (PAA, 35 $\%$ in $\mathrm{H}_{2} \mathrm{O}$ ) with a molecular weight of $100,000 \mathrm{~g} \mathrm{~mol}^{-1}$, m-phenylenediamine (MPD) with $99 \%$ purity, trimesoyl chloride (TMC) with $98 \%$ purity, Brilliant Blue R (pure, $825.97 \mathrm{~g} \mathrm{~mol}^{-1}$, negative charge), and Disperse red 1(Dye content $95 \%, 314.34 \mathrm{~g} \mathrm{~mol}^{-1}$, neutral), sodium sulfate (Na2SO4), and calcium chloride $(\mathrm{CaCl} 2)$. The following solvents were purchased from Fisher Scientific and were used as received: toluene, acetone, ethanol, n-hexane, methanol, and isopropanol. Molecular 
sieves ( $3 \mathrm{~A}^{\circ}$, Acros Organics) were used to dehydrate toluene and acetone. Deionized (DI) water from the Millipore milli-Q purification system with the resistance of $18.2{\mathrm{M} \Omega \mathrm{cm}^{-1}}^{-1}$ was used for all experiments. Silicon wafers (single-side-polished (SSP), $<100>$ orientation, type-P, dopant-B, 25.4 and $50.8 \mathrm{~mm}$ diameter) were purchased from University Wafers. Polyacrylonitrile support (PAN50) was purchased from Sepro Membranes Inc. Aluminum oxide membrane filters of diameter $25 \mathrm{~mm}$ with pore size $200 \mathrm{~nm}$ were purchased from Sterlitech Inc. and used as a support for SEM. Flat sheet commercial membranes, SW30HR, were purchased from Dow FILMTEC.

\subsection{Characterization methods}

\subsubsection{Scanning electron microscopy (SEM)}

The morphology of nanofilms fabricated was investigated by using a high-resolution SEM, JOEL 6500 field emission. The freestanding nanofilms were transferred onto AAO or HPAN supports for SEM imaging. For cross-sectional images, thin film membranes were prepared by freeze-fracturing in liquid nitrogen and subsequently drying in the air. Samples were sputter-coated with platinum (15 $\mathrm{nm}$ thickness) before SEM imaging. The thickness measurements of nanofilms were analyzed from cross-sectional SEM views using ImageJ software. The freestanding CNDs nanofilm was placed on a carbon tape using a lasso as shown in Figure 1f. To obtain Figure 2h, the synthesized TFC membrane (nanofilms directly synthesized in HPAN) was freeze-fractured in liquid nitrogen followed by peeling the CNDs layer from HPAN using a double-sided tape. The peeled CNDs layer was then placed on a carbon tape for SEM imaging.

\subsubsection{Transmission electron microscopy (TEM)}

The morphology of synthesized CNDs and CNDs nanofilms was investigated using TEM, JEOL 2100 , operated at $200 \mathrm{kV}$. Few drops of a dilute CNDs solution in toluene were drop-casted on a 
copper grid for TEM imaging. A small piece of freestanding nanofilm was transferred to a copper grid for TEM characterization.

\subsubsection{Atomic force microscopy (AFM)}

The fabricated films on silicon wafers were characterized using AFM microscope (Dimension Icon with ScanAsyst) to measure the thickness and surface roughness values. A sampling resolution of 512 points per line and a speed of $1 \mathrm{~Hz}$ were used. Bruker 'Nanoscope Analysis' analysis software was used to measure surface roughness and film thickness. Surface roughness is presented as average roughness $(\mathrm{Ra})$, root-mean-square roughness $(\mathrm{rms})$. The freestanding nanofilms were formed on oxidized silicon wafer for thickness measurements using AFM. The sample was scratched using a razor blade, and the change in height from the film surface to the bare wafer surface is reported as nanofilm thickness. Gwyddion 2.38 SPM data visualization software was used to process the AFM images (Figure 2f).

\subsubsection{Contact angle measurement}

Surface wettability of the synthesized nanofilms was investigated using water contact angle measurements with a drop shape analysis system (DSA 100, KRUSS, Germany). A drop of water (about $25 \mu \mathrm{l}$ ) was deposited on the membrane surface. The contact angle was measured using a circle fitting method by ImageJ software. The average value of contact angles with standard deviation are presented.

\subsubsection{X-ray photoelectron spectroscopy}

The CNDs nanofilms were synthesized on an oxidized silicon wafer for XPS characterization. The XPS experiments were conducted at the Integrated Microscopy Center, the University of 
Memphis using a Thermo scientific K-alpha XPS system equipped with a monochromatic X-ray source at $1486.6 \mathrm{eV}$, corresponding to the $\mathrm{Al} \mathrm{K}_{\alpha}$. A spot size of $400 \mu \mathrm{m}^{2}$ was used. Measurements were conducted in the constant analyzer energy mode. The survey spectra were collected at pass energy of $200 \mathrm{eV}$ over a range of $0-1300 \mathrm{eV}$. The high resolution (HR) core spectra were collected at a $40 \mathrm{eV}$ pass energy and an energy step size of $0.1 \mathrm{eV}$, and using an average of 20 scans. HR scans of $\mathrm{C} 1 \mathrm{~s}, \mathrm{O} 1 \mathrm{~s}$, and N1s have also been conducted. XPS data processing was performed using CasaXPS software.

\subsubsection{Ultraviolet-visible (UV/Vis) spectroscopy}

An Agilent $8510 \mathrm{UV} / \mathrm{Vis}$ spectrometer was used to record the absorption spectra of dye solutions. Solutions were loaded to $1 \mathrm{~mm}$ path length quartz cells. A calibration curve was obtained to predict the concentration of the dye solution by conducting its absorption spectra. 
i. Spin coat 3 layers of PAA
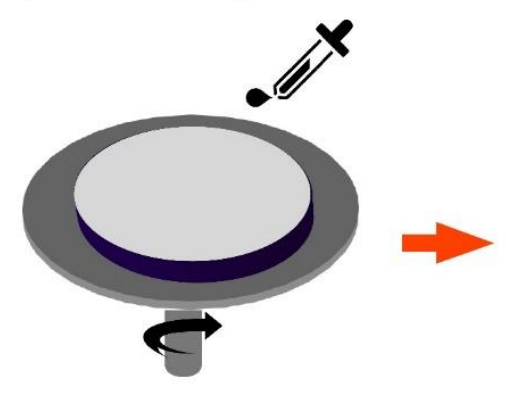

iv. Transfer the active layer on different support

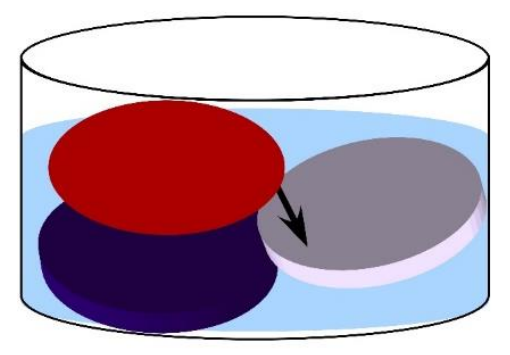

ii. Spin coat 30 active layers
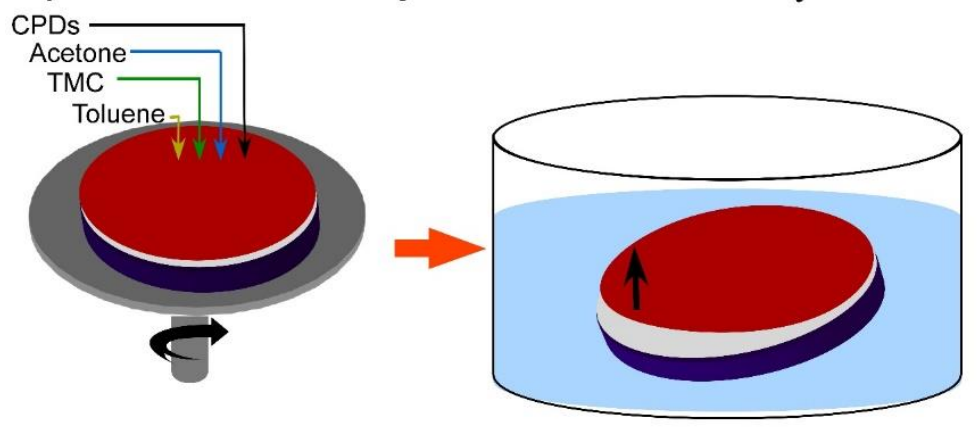

iv. Install the thin film in the filter apparatus

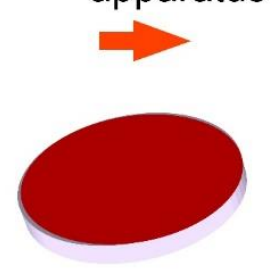

iii. Solve PAA layer

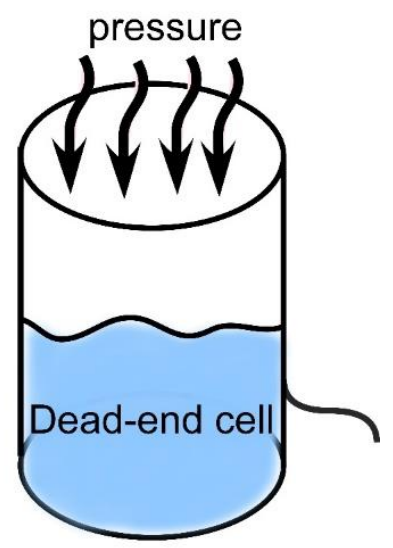

Figure S1. Schematic of fabrication of freestanding nanofilm using a water-soluble sacrificial layer. i) 3 layers PAA solution is spin-coated on an oxidized silicon wafer at $500 \mathrm{rpm}$ for 45 seconds to ensure full coverage of silicon wafer surface then at $2000 \mathrm{rpm}$ for 20 seconds followed by drying the surface using an air gun and a hot plate; ii) 30 layers of CNDs nanofilm was formed on the surface via depositions of CNDs solution in toluene, acetone, TMC in toluene, and toluene, respectively. One cycle of CNDs solution, acetone, TMC, and toluene yields one layer of CNDs nanofilm, herein, 30 layers of CNDs nanofilm was formed. iii) After the formation of CNDs nanofilm, the sample was dipped in a water bath to obtain freestanding CNDs nanofilm. The PAA layer dissolved in water gradually, so enough time should be given in this step to minimize the defects and wrinkles formation in freestanding film. iv) The floating freestanding CNDs nanofilm can be transferred to different supports. Enough time should be given to dry, letting the CNDs nanofilm physically attach to the support layer. iv) The resultant thin film membrane can be tested 
in a filtration set-up (i.e. dead-end cell, or vacuum filtration setup) or used for characterization studies.

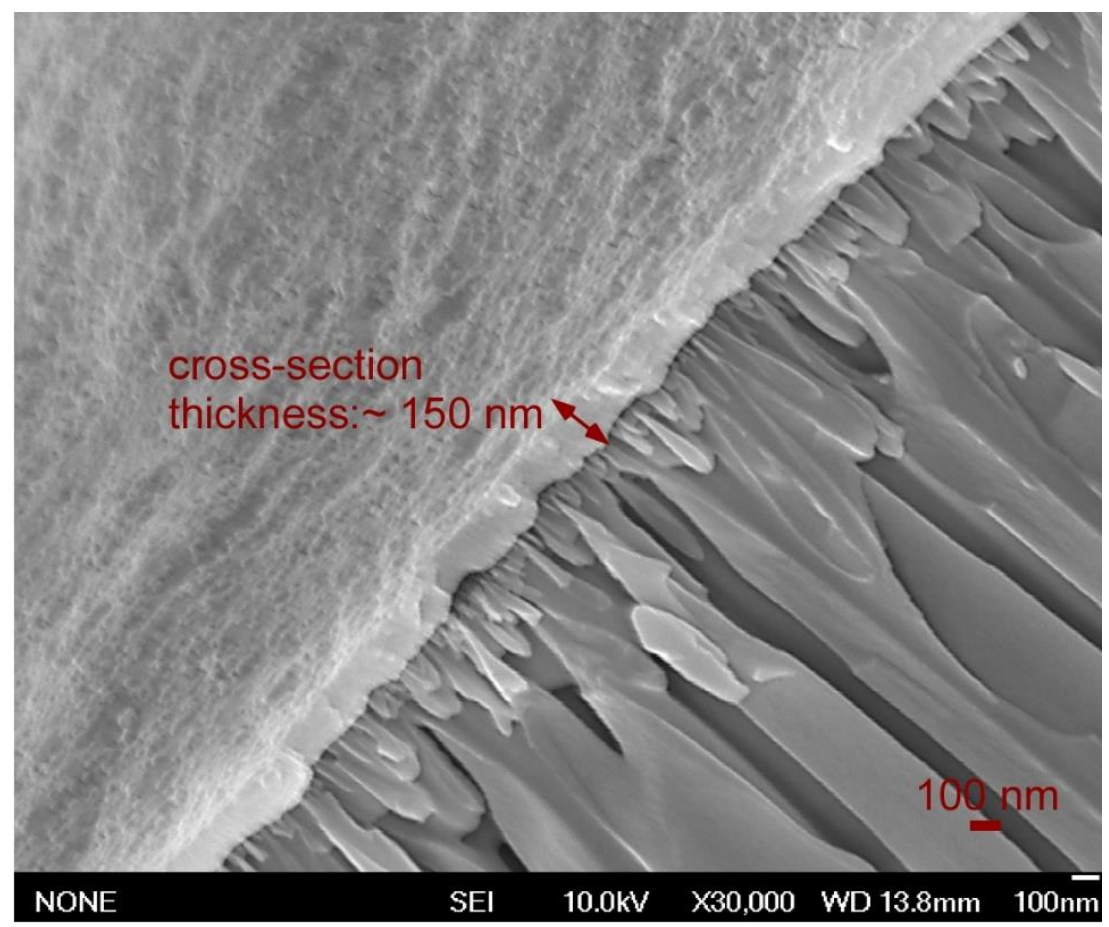

Figure S2. SEM cross-section image of freestanding CNDs nanofilm transferred onto porous alumina support layer. 


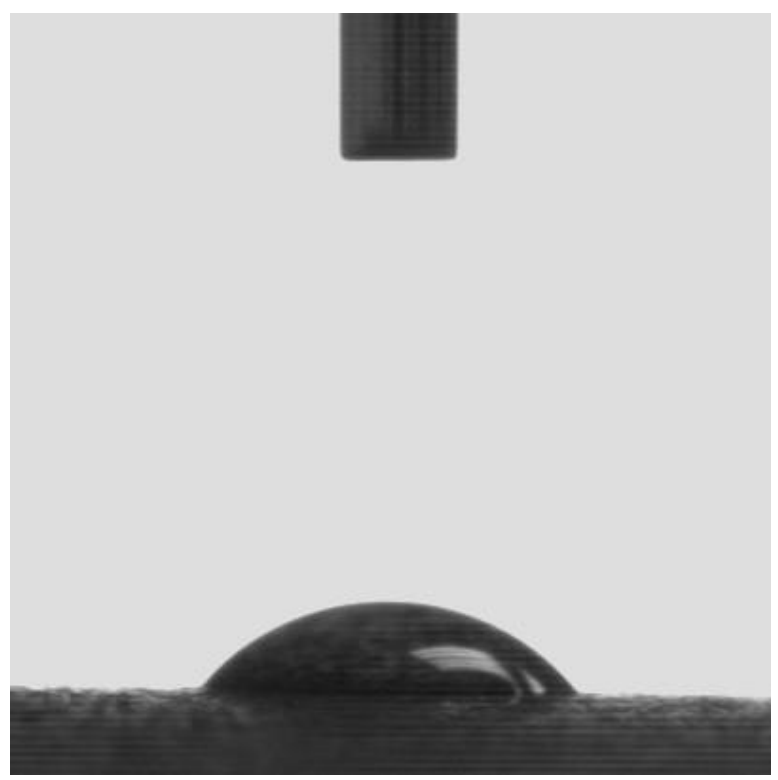

Figure S3. The water contact angle of CNDs nanofilm formed on a silicon wafer.

2. Methods

2.1. XPS data for CNDs powder and CNDs nanofilms 

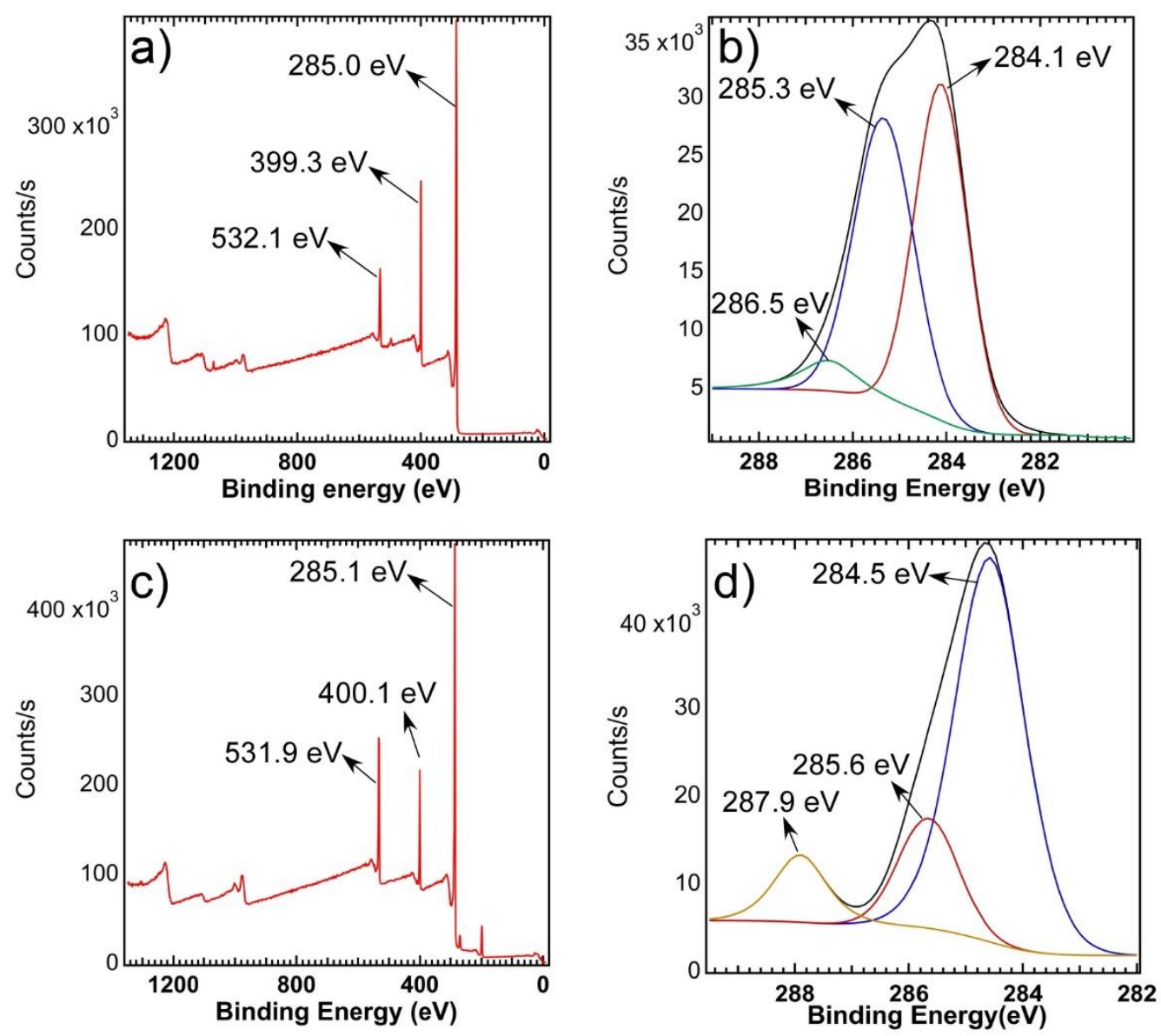

Figure S4. a) XPS long scan (LS) data of CNDs powder; b) High-resolution C1s spectrum of CNDs powder; c) Long scan spectra of CNDs nanofilm, d) high-resolution C1s spectra of CNDs nanofilm.

\subsection{OSN performance of CNDs films}

The organic solvent permeance and dye rejections (in methanol) were conducted at room temperature in a lab-scale dead-end system (Sterlitech, U.S.A). The system contained a pressure feed vessel where compressed nitrogen was used to apply the pressure. Data reported was collected after a 0.5 -hour operation. For the DF-CNDs, 20 bar pressure was used and for FSCNDs 10 bar pressure was used. For dye rejection studies, brilliant blue (BB) and disperse red (DR) dye solution in methanol with a concentration of $20 \mathrm{mg} \mathrm{L}^{-1}$ was used. The rejection value 
was calculated from the UV-vis absorption peak in the feed and permeate. The calibration plots for $\mathrm{BB}$ and $\mathrm{DR}$ were developed by relating peak intensity and their known concentration in methanol. In addition to $\mathrm{BB}$ and $\mathrm{DR}$, solutes of $\mathrm{CaCl}_{2}$ and $\mathrm{Na}_{2} \mathrm{SO}_{4}$ were used in aqueous solutions for plotting the rejection against molecular weight. Equation 1 shows the rejection $(\mathrm{R})$ calculation:

$$
R \%=\left(1-\frac{C_{p}}{C_{f}}\right) \times 100 \%
$$

$\boldsymbol{C}_{\boldsymbol{p}}$ and $\boldsymbol{C}_{\boldsymbol{f}}$ were the dye concentrations in the premeate and feed, respectively.

The solvents flux $J_{w}$ was determined as

$$
J_{w}=\frac{V}{t A}
$$

$\mathrm{V}$ is the permeate volume collected over time, $\mathrm{t}$. A is he membrane effective area. The solvent permeance was determined as solvent flux over applied pressure (bar).

Table S1. Hansen solubility parameter $(\delta)$ and the physical properties of the organic solvents used in this study ${ }^{1}$

\begin{tabular}{lllll}
\hline Solvents & Methanol & Ethanol & Isopropanol & Toluene \\
\hline Molar diameter, $\mathrm{d}_{\mathrm{m}}(\mathrm{nm})$ & 0.51 & 0.57 & 0.62 & 0.70 \\
Viscosity $\left(\times 10^{-3} \mathrm{~Pa} . \mathrm{s}\right)$ & 0.49 & 1.17 & 2.1 & 0.52 \\
Hansen solubility parameter & 12.3 & 8.8 & 6.1 & 1.4 \\
(dipole forces), $\delta_{\mathrm{p}}$ & & & &
\end{tabular}




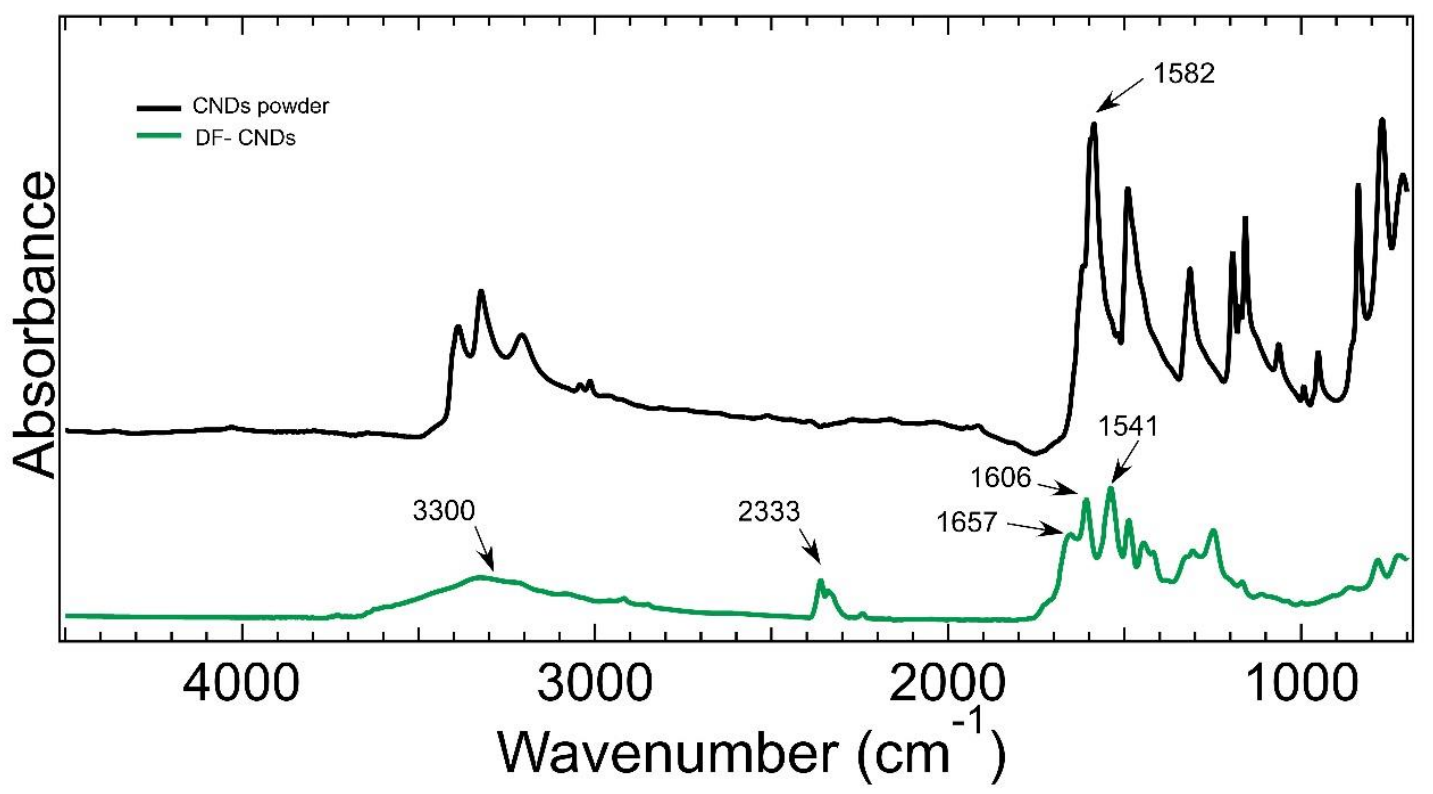

Figure S5. FTIR spectra for CNDs powder (black line) and DF-CNDs membrane (green line)

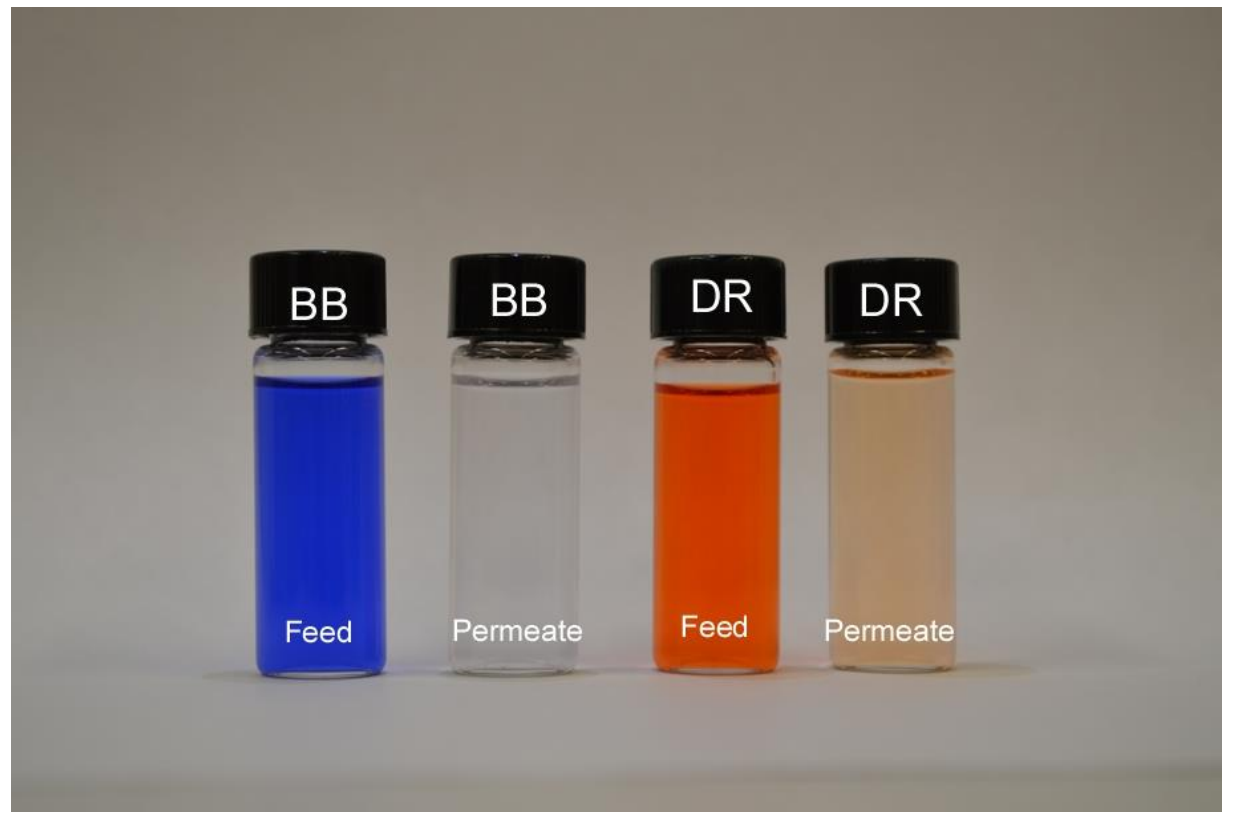

Figure S6. Brilliant blue (BB) and disperse red (DR) feed and permeate solutions for DF-CNDs membranes tested in dead-end cell at 20 bar pressure. 


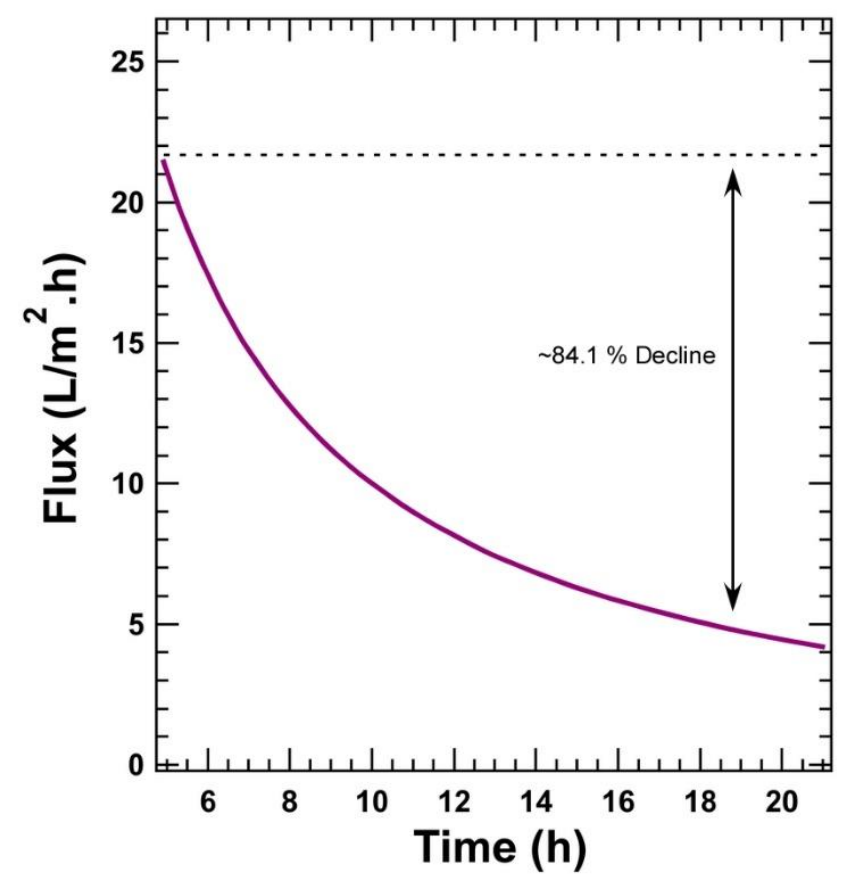

Figure S7. OSN performance for SW30HR membrane as a function of time. Pure methanol permeance through an SW30HR commercial membrane displays about $84.1 \%$ decline in flux in $20 \mathrm{~h}$. The dead-end test has been performed at $10 \mathrm{bar}$ and at ambient temperature. 


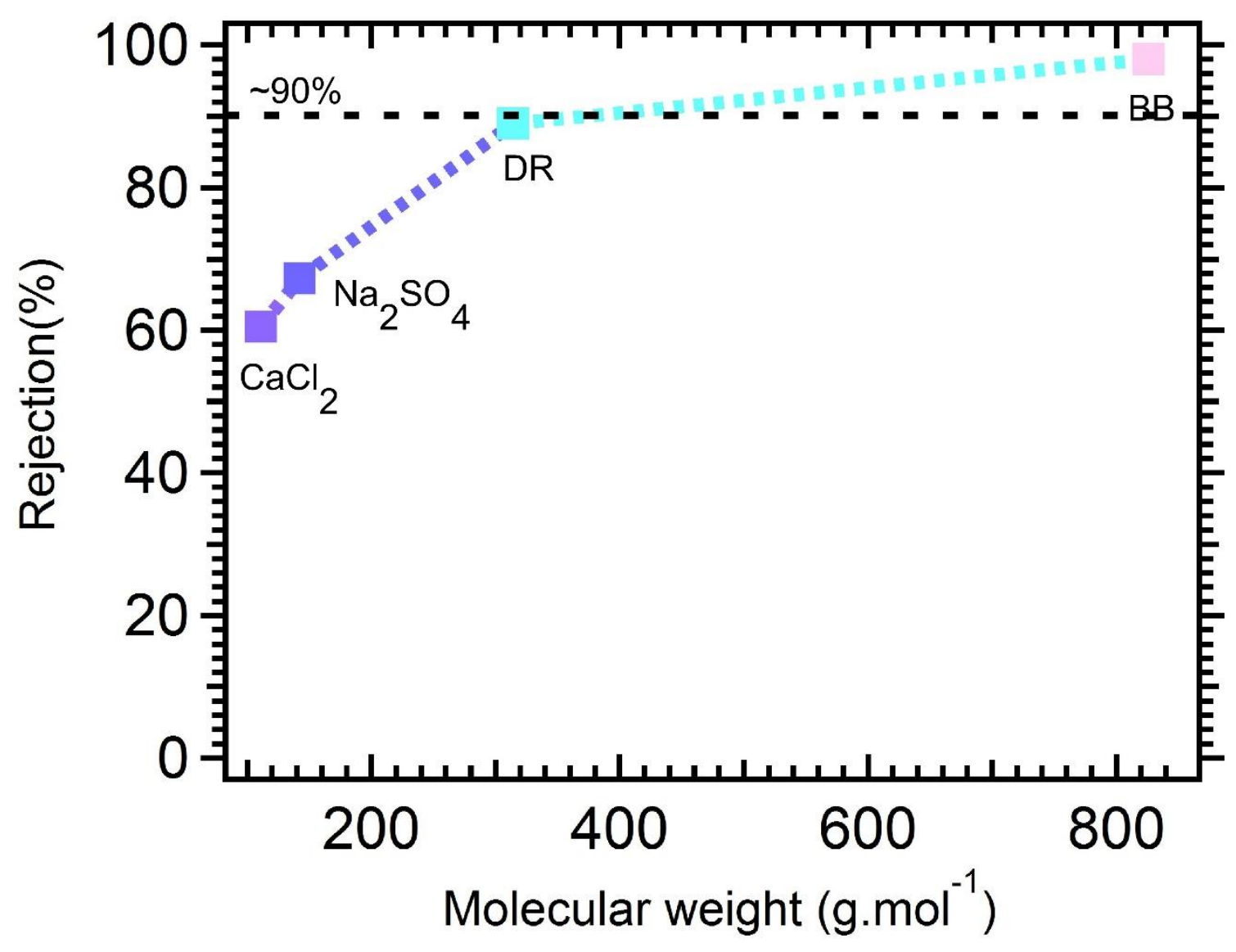

Figure S8 Rejection data for DF-CNDs. Solutes and dyes with different molecular weights were used. Feed pressure: 20 bar, feed concentration: $20 \mathrm{mg} \mathrm{L}^{-1}$, and stirring speed of $500 \mathrm{rpm}$. Two dyes of $\mathrm{BB}$ and $\mathrm{DR}$ were solved in methanol. Aqueous solutions of calcium chloride $(\mathrm{Mw} \approx 110.9$ $\left.\mathrm{g} \mathrm{mol}^{-1}\right)$ and sodium sulfate $\left(\mathrm{Mw} \approx 142.04 \mathrm{~g} \mathrm{~mol}^{-1}\right)$ were used. The dashed line shows $90 \%$ rejection, and the corresponding molecular weight is used as the molecular weight cut off value for this membrane. 


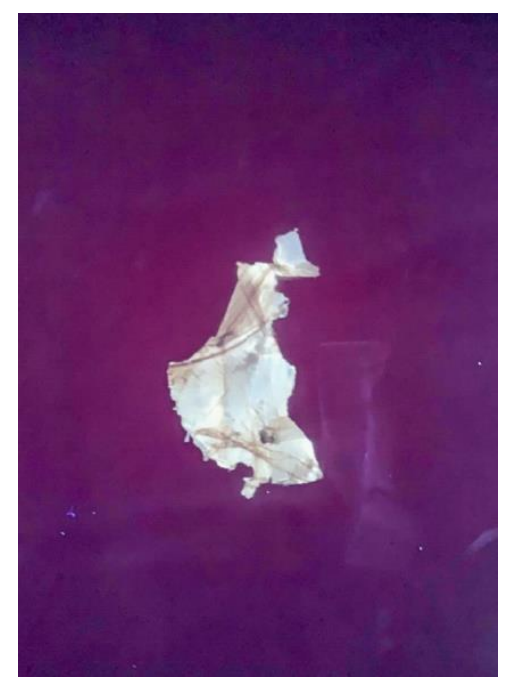

Figure S9. Image of freestanding CNDs nanofilm under UV light. The nanofilm has been lift-off from silicon wafer after being fabricated by LbL assembly.

References:

(1) Karan, S.; Jiang, Z.; Livingston, A. G. Sub-10 Nm Polyamide Nanofilms with Ultrafast Solvent Transport for Molecular Separation. Science 2015, 348 (6241), 1347-1351. 